\title{
Quand les entreprises produisent du capital social
}

Étude d'un cas de coopération interentreprises

When Businesses Produce Social Capital. A Case Study of Inter-Business

Cooperation

Eric Forgues, Omer Chouinard et Danièle Courchesne

\section{OpenEdition}

Journals

Édition électronique

URL : http://journals.openedition.org/economierurale/2295

DOI : 10.4000/economierurale.2295

ISSN : 2105-2581

\section{Éditeur}

Société Française d'Économie Rurale (SFER)

Édition imprimée

Date de publication : 1 juin 2009

Pagination : 19-34

ISSN : 0013-0559

Référence électronique

Eric Forgues, Omer Chouinard et Danièle Courchesne, "Quand les entreprises produisent du capital social », Économie rurale [En ligne], 311 | Mai-juin 2009, mis en ligne le 05 mai 2011, consulté le 01 mai 2019. URL : http://journals.openedition.org/economierurale/2295 ; DOI : 10.4000/ economierurale.2295 


\section{Quand les entreprises produisent du capital social Étude d'un cas de coopération interentreprises}

Eric FORGUES • Institut canadien de recherche sur les minorités linguistiques, Université de Moncton, Canada

Omer CHOUINARD et Danièle COURCHESNE • Université de Moncton, Canada

\section{Introduction}

$\mathbf{L}^{\mathrm{c}}$ e présent article fait suite à une étude exploratoire réalisée en 2006-2007 sur l'association de plusieurs boucanières dans le village de Cap-Pelé et ses environs dans le sud-est du Nouveau-Brunswick ${ }^{1}$. Les acteurs de cette industrie locale éprouvaient plusieurs difficultés en raison de la forte concurrence qui s'exerçait entre les boucanières, entraînant même plusieurs conflits entre les familles de cette petite communauté côtière. L'idée de former une association n'est pas nouvelle. Plusieurs tentatives avaient été faites vainement dans le passé. En 2003, le propriétaire d'une boucanière relance le projet de créer une association entre les boucanières en faisant appel aux services d'une personne extérieure à l'industrie du hareng fumé et au village qui, de son propre aveu, ne connaissait rien à ce domaine et ne voyait pas comment il pouvait mener à bien ce projet $t^{2}$. Pourtant, non seulement a-t-il réussi à créer une association regroupant 18 boucanières sur 25 , Les exportations de Harengs Cap-Pelé Inc, mais l'association existe toujours plus de cinq années après sa création. Au-delà de la perception

\footnotetext{
1. Pour respecter l'usage établi dans la région du sud-est du Nouveau-Brunswick, nous appelons boucanière l'usine de harengs fumés et séchés. En fait, les boucanières fument et salent plusieurs types de produits marins (gaspareau, morue, maquereau). Le village de Cap-Pelé compte près de 279 habitants en 2006, soit 750 familles (données de Statistique Canada). Il est situé sur la côte de l'Atlantique au Canada.

2. Entrevue réalisée avec les chercheurs.
}

selon laquelle la réussite de ce projet s'explique par l'intervention de cette personne, nous avons voulu comprendre comment eston passé d'une situation de forte concurrence, qui menait littéralement plusieurs boucanières à la faillite, à une situation de coopération. Quels éléments permettent de comprendre cette transition sociale ? $\mathrm{Ce}$ type de question est au cœur de notre démarche de recherche qui intègre d'emblée l'économie à la vie sociale, mais qui y perçoit également des éléments de production du social (Lévesque et al., 2001).

Pour comprendre les éléments fondamentaux de cette transition sociale, les concepts de capital social et de coopération interentreprises paraissent particulièrement éclairants. Nous présenterons donc, dans un premier temps, ces concepts et quelques éléments pertinents de la littérature, avant de rendre compte des divers éléments sociaux qui contribuent à la constitution du cas de coopération interentreprises à l'étude, tels que l'intérêt, les règles, les symboles et la confiance. L'intérêt d'un cas semblable réside dans le fait qu'il constitue un observatoire de choix dans l'étude de la transition entre une situation sous-socialisée et une situation socialisée, pour reprendre la terminologie de Granovetter (1985).

Pour les besoins de notre analyse, nous avons réalisé des entrevues semi-dirigées avec huit propriétaires de boucanière membres du regroupement, avec le directeur de l'agence de mise en marché et avec un propriétaire de boucanière demeuré indépendant. Nous avons également consulté des études sur l'industrie du hareng fumé et 
l'histoire de la région, ainsi que des archives fournies par les répondants. L'analyse de ces entrevues nous a permis de dégager les éléments constitutifs de l'association entre les boucanières et de mieux comprendre le mécanisme de transition entre une situation de concurrence et une situation de coopération. Cette étude de cas exploratoire permet d'élaborer des pistes menant à la définition des modèles de coopération interentreprises grâce auxquels les entreprises placées dans une situation fortement concurrentielle sont mieux à même d'affronter les défis qui se posent à elles.

\section{Capital social et coopération interentreprises}

La sociologie économique (Lévesque et al., $o p$. cit.) enseigne que l'économie est intégrée dans la société et que sa forme et son développement sont déterminés par des facteurs sociaux. Ainsi, l'économie ne fonctionne pas en vase clos au sein de la société. Dans la même veine, la sociologie des entreprises (Sainsaulieu, 1992) montre clairement l'ancrage social des entreprises de même que leur contribution à la production du social. C'est un article de Granovetter (1985) qui a réanimé la sociologie économique en montrant l'inscription sociale des pratiques économiques. Dans un article antérieur (Granovetter, 1973), l'auteur avait illustré le rôle que jouent les liens faibles sur le marché du travail. Dans cette continuité, la sociologie économique a tôt fait par la suite d'enrichir la littérature sur le capital social en soulignant la fonction qu'assument les réseaux et les ressources qu'ils mobilisent dans le développement et les pratiques économiques. Si l'idée selon laquelle les liens sociaux, les normes, les valeurs et la confiance contribuent aux pratiques économiques n'est pas neuve, le fait de considérer ces dimensions comme un capital qui stimule le développement économique est nouvelle (Ponthieux, 2003 ; Grewe, 2003). Par conséquent, le capital financier et le capital humain ne peuvent être pleinement maximisés sans le capital social (Mubangizi, $2003)^{3}$.

Plusieurs définitions du capital social existent ; certaines insistent sur les liens sociaux, d'autres, sur les ressources échangées au sein des réseaux ou sur l'engagement social ${ }^{4}$. Dans notre perspective, le capital social désigne les réseaux et les liens sociaux grâce auxquels un individu ou une communauté peut accéder à des ressources (économiques, politiques, culturelles ou humaines) nécessaires à l'atteinte de ses objectifs. En limitant ainsi la définition du capital social à la constitution de réseaux et de liens sociaux, nous augmentons grandement le pouvoir explicatif du concept (Judge, 2003) $)^{5}$ Le capital social renvoie autant à des dimensions qualitatives que quantitatives. Ce concept ne désigne pas seulement des relations ou des réseaux dont dispose une communauté ou un individu pour réaliser certaines activités ; il comprend aussi des types de relations, qui se différencient selon leurs valeurs constitutives, les règles de réciprocité, les sentiments d'obligation, la confiance et l'engagement communautaire des individus.

Généralement isolé de son contexte social par les théories utilitaristes ou économistes, l'agent économique se trouve réinscrit dans la société et ses réseaux d'appartenance par les théories sur le capital social. Si la littérature tend à mettre en évidence la contribution du capital social dans une série de phénomènes sociaux ou économiques, elle est peu encline à étudier les conditions de production du capital social. Comment se construit la confiance, les liens, les rapports de réciprocité entre des agents économiques et, en définitive, leur sentiment d'appartenance à un groupe ou à une communauté ?

3. In Mubangizi, page 142.

4. Sur les différentes conceptions du capital social, voir Forgues (2004 et 2007).

5. In Judge, page 11. 
Notre étude d'un cas de Coopération interentreprises (CIE) ne se limite pas à mettre en lumière la contribution du capital social dans les pratiques économiques d'une communauté côtière rurale. Elle précise les éléments qui permettent de comprendre comment se forme le capital social. C'est dans cette perspective que nous examinons un cas de CIE qui réunit les conditions sociales requises pour créer des alliances entre les entreprises à l'étude, manifestant ainsi une innovation sociale pour résoudre des difficultés économiques. L'analyse qui suit présente des éléments de compréhension de cette transition vers une forme de CIE, tout en reconnaissant qu'il s'agit d'une étude exploratoire qui devrait être approfondie pour mieux éclairer le rôle que joue le contexte socioculturel et sociohistorique dans cette transition. Elle comporte néanmoins l'avantage de révéler des facteurs sociaux et symboliques qui ne peuvent pas se réduire à la logique de l'intérêt ni à la rationalité économique des acteurs si on veut saisir dans sa globalité le mode de transition vers la CIE.

Selon la littérature, plusieurs facteurs peuvent inciter les entreprises à s'associer. «Des entreprises concurrentes dans un même secteur peuvent être conduites à envisager des stratégies collectives et à gérer ainsi leurs interdépendances [...] en unissant leur destin » (Yami, 2003) ${ }^{6}$. Pour Bellon et Niosi (2001), l'objectif consiste à réduire l'incertitude : «In cooperative agreements, the goal is only to reduce uncertainty, risk and the natural myopia of economic agents $»^{7}$. L'incertitude est généralement associée à l'impossibilité de prévoir les comportements d'autres acteurs dans un contexte où ces comportements influent sur l'environnement des entreprises. Cette incertitude s'accroît lorsque chaque acteur poursuit un intérêt qui est le sien sans s'allier aux autres acteurs, les plaçant dans des situations

6. In Yami, page 166.

7. Cf. page 73 . de compétition et de concurrence. Elle peut être réduite lorsque les acteurs coordonnent leurs activités. L'intérêt que présente la CIE pour les acteurs est qu'elle leur permet d'atteindre pleinement leur objectif. Dans notre cas, la réduction de l'incertitude visait à mieux planifier la production et à stabiliser les prix et les ventes.

Bellon et Niosi (op. cit.) précisent que, dans les ententes de CIE :

- les entreprises conservent leur autonomie, - le partage des capitaux engage les ressources des partenaires,

- l'objectif à atteindre demande temps, innovation, stratégie de développement.

Les accords passés entre les entreprises se concrétisent dans la forme d'un contrat qui scelle leur alliance et définit à la fois sa portée, les conditions et les règles à respecter de même que ses limites. Selon Avadikyan et al. (2001) « the main function of rules which have as their ultimate goal the allocation of resources will be to urge the partners to "behave properly", using mechanisms of extrinsic incentives such as sanction/reward or control set ups. Their degree of codification is considered to be high $»^{8}$. Le contrat permet d'orienter les comportements des acteurs de façon à éviter qu'ils se nuisent les uns les autres. Toutefois, il ne suffirait pas seul à garantir ce résultat. Certains auteurs estiment que la confiance mutuelle provient, entre autres, de l'intégration sociale de leurs activités économiques. En fait, plus la confiance est réciproque, moins le contrat formel doit être précis et moins il intervient dans la réduction de l'incertitude et des comportements opportunistes individuels. «Social embeddedness reduces the necessity of contractual commitments through the emergence of initial trust and the availability of (implicit) threats » (Blumberg, 2001) ${ }^{9}$.

8. In Avadikyan et al., page 1449.

9. In Blumberg, page 829. 
Les liens sociaux, les règles informelles de réciprocité, la confiance, la réputation, la parole donnée et le serrement de mains forment un capital social qui permet aux acteurs de coordonner leurs activités. L'intégration des activités économiques par la voie de liens informels apparaît comme un des facteurs de régulation des rapports sociaux. Si certains comportements ne sont pas admis et qu'ils sont répétés par certains acteurs, ces derniers risquent à la longue d'être exclus du réseau d'échanges économiques. Les occasions favorables diminuant d'autant, ils se trouvent forcés de réorienter leurs comportements. "Thus in summary, informal institutions [...] allowed actors credibly to commit to each other and thus to trust each other enough to maintain relationship that involved a high level of reciprocity and gift exchange $\gg$. (Farrell, 2005) ${ }^{10}$. Outre les règles informelles, le contrat qui scelle la CIE permet également de réguler les comportements des membres de l'alliance. Ainsi, les normes informelles et les règles formelles assurent l'existence de l'alliance. À défaut de tels mécanismes de régulation, les entreprises poursuivent isolément l'accroissement de leur seul intérêt et ne parviennent pas à travailler de concert. C'est vers cette situation que se dirigeaient les boucanières avant 2003 .

Comme le mentionnent Guillouzo et Thépaut (2004), la CIE offre un cadre organisationnel qui possède plus de flexibilité qu'une organisation corporative hiérarchique tout en réduisant les risques et les incertitudes d'une situation de marché. Elle permet en outre aux entreprises de se concentrer sur leurs forces et de confier certaines tâches aux partenaires ou encore à une structure issue de l'alliance. D'ailleurs, la particularité de l'entente de CIE entre les boucanières a consisté en la création d'une association d'entreprises. Les boucanières se concentrent ainsi sur la production, alors que le groupe créé se

10. In Farrzell, page 474. charge de la commercialisation de la production $^{11}$. De plus, la CIE permet aux entreprises de mieux se positionner sur le marché.

Rappelons que la CIE puise d'abord à la vie associative en conciliant utilitarisme et réalité sociocommunautaire. Elle puise à l'utilitarisme, mais "le marché se révèle insatisfaisant », alors que "la vision de l'association comme poursuite des intérêts de ses membres, au lieu de constituer une réponse définitive sur l'origine associative, renvoie à un questionnement sur les modes de construction de cet intérêt » (Laville et Sainsaulieu, 1997) ${ }^{12}$. Elle puise enfin à « la réalité sociocommunautaire » dans un contexte où le libéralisme menace le lien social. L'association peut ainsi dépasser l'approche « en termes purement contractuels » et être de « l'ordre de la construction communautaire » qui s'inscrirait dans « un espace démocratique revendiquant liberté et égalité des membres ». (idem, 1997) ${ }^{13}$. Ajoutons que, selon ces auteurs, l'association est « source de solidarité » en ce sens que l'individu est amené « à compter sur autrui » Ainsi, on peut parler du fait associatif, qui réside dans "l'affirmation $d u$ lien social, et sa diversité, qui s'explique par la multiplicité des ressorts dont il peut procéder».

Tout en concédant qu'il s'agit d'une première étude exploratoire, nous nous proposons d'analyser la notion de CIE, à travers le cas des boucanières de la région de Cap-Pelé, en reconnaissant d'entrée de jeu la complexité d'un tel arrangement social qui mobilise l'intérêt des acteurs, le cadre contractuel et les règles formelles connexes, sans négliger les facteurs symboliques et normatifs qui président au maintien de cette coopération.

11. Nous verrons plus loin que les responsabilités du groupe se sont accrues par la suite.

12. In Laville et Sainsaulieu, pages 44-45.

13. In Laville et Sainsaulieu, page 51, puis pages 62 et 65 pour les citations suivantes. 


\section{Coopération interentreprises entre des boucanières}

\section{Contexte historique}

La région de Cap-Pelé a représenté longtemps une destination privilégiée pour les harengs venus frayer au printemps le long de la côte. Le Cap-Pelé s'appelait d'ailleurs cap Hareng au XVII siècle (Brun et LeBlanc, 2005). On trouve des indications de l'existence d'une boucanière dans les années 1840 ; les premières boucanières commerciales s'établissent dans les années 1910 dans la région de Cap-Pelé. Même si les Acadiens de la région ont acquis avec le temps un savoir-faire qui leur est propre, ce sont des anglophones de Chimougoui (Shemogue) et de Grand Manan dans la baie de Fundy au sud-ouest du Nouveau-Brunswick qui leur ont enseigné les techniques de production commerciale du hareng. L'industrie se développe assez rapidement, passant de deux boucanières dans les années 1910 à une vingtaine dans les années 1950. C'est le village de Bas-Cap-Pelé qui concentre le plus de boucanières, mais certaines s'installent également dans les villages de Trois-Ruisseaux et de Dupuis Corner. En 1986, la région compte 25 boucanières.

Notons qu'une forte inégalité existe dans la production des boucanières : une seule boucanière contribue au tiers de la production totale de 19 boucanières et elle produit plus que les 11 boucanières les moins productives réunies. Les trois boucanières les plus productives contribuent à l'équivalent de la production de 16 boucanières réunies. Selon une étude réalisée en 2000 par une société conseil (LeBlanc, 2000), 31 producteurs géraient 25 boucanières. L'industrie dans la région de Cap-Pelé embauchait 900 employés à temps partiel, représentant 438 emplois à temps plein. Durant les cinq années antérieures à l'étude, on estime que la production annuelle est de 2 millions de boîtes (à savoir 18000 tonnes américaines). Cependant, une étude de Pêches et Océans Canada (1987) révèle qu'en 1986 les 25 usines produisaient 1 million de boîtes de produits marins (soit
9000 tonnes), doublant leur production en quelques années et générant près de 7 millions \$ en 1986. Selon l'étude de LeBlanc (op. cit.), la valeur de la production est égale à 40 millions \$. En 1986, la valeur de la production s'élevait à 15 millions $\$$, ce qui représente une hausse de $160 \%$. Enfin, soulignons que la région produit plus de $90 \%$ de la production totale de harengs fumés au Canada.

Le marché des produits marins fumés est demeuré assez stable jusque dans les années 1970, mais, par la suite, l'arrivée d'un groupe d'acheteurs rend plus vive et plus acharnée la compétition entre les boucanières (Co-fish consultants, 1985) ${ }^{14}$ Un rapport prévoit que, plus il y aura de boucanières sur le marché, " there will be less coordination of effort, more price competition and the price level will decline $\gg(\text { idem })^{15}$. Aussi recommande-t-il aux exploitants de mieux coordonner leurs activités afin d'atténuer cette compétition nuisible à l'ensemble de l'industrie. Cette recommandation est formulée au moment où une association existe depuis 1984 . Toutefois, plusieurs boucanières ne respectent pas les conditions de l'entente, notamment sur les prix de vente (idem). L'association ne prévoyait pas de moyens contraignants, tels qu'une amende, pour s'assurer que les membres se conforment à l'entente. C'est sur une base volontaire qu'on demandait aux boucanières de passer par la coopérative pour effectuer leurs ventes. Les ratés de l'association apparaissent clairement quelques années après sa création. Au moment de l'étude, en 1985, les membres de l'association en étaient encore à s'interroger sur la loyauté de chacun et à se buter contre la phase de l'observation mutuelle. D'après ce rapport, la

14. Nous présenterons les principaux éléments d'analyse de ce rapport et ses recommandations, puisque ce sera le modèle adopté en 2003 par les boucanières.

15. In Co-fish consultants, page 157. 
solution la plus acceptable, et celle qui a le plus de chance de réussir, serait que toutes les boucanières s'engagent à créer en copropriété une agence de mise en marché. L'agence appartiendrait aux membres de l'association, elle serait composée d'un conseil de direction auquel siégeraient des membres du groupe, elle ferait la vente et la promotion des produits et elle conserverait un pourcentage des ventes pour couvrir le coût de l'exploitation. L'agence contrôlerait les taux et les quantités de production à l'aide quotas (idem). Elle serait chargée à la fois de vendre le poisson et de créer un logo pour l'entreprise (idem).

Il est intéressant de noter que le rapport souligne l'importance et le défi que représente le fait de dépasser un comportement fondé sur l'intérêt personnel : "Processors will have to forego their present rather egoistic approach to marketing $(\text { idem })^{16}$. L'auteur du rapport croit que l'embauche d'un gestionnaire permettrait de renforcer les relations entre l'agence et ses membres (idem) Selon lui, une association entraînerait des effets bénéfiques dans la communauté en réduisant la méfiance entre les gens, en invitant les boucanières à prendre conscience de la nécessité de se concerter pour faire face à des difficultés communes et en favorisant la volonté de travailler ensemble (idem). L'auteur du rapport est conscient de la concurrence que se livrent les boucanières et reconnaît qu'une démarche collective peut prendre du temps à s'implanter : "... it will take time to prove to the "individualists", in their ranks that the collective way of doing things can be the best way » (idem).

\section{Vers une coopération interentreprises}

Le projet visant la création d'une association de boucanières est donc relancé en 2003, alors qu'un propriétaire approche un médiateur reconnu pour lui proposer de mener cette initiative en tant que gérant.

16. In Co-fish consultants, pages 197-198.
Cette personne provenait non seulement de l'extérieur de l'industrie, mais également de l'extérieur des limites du village de Cap-Pelé. Ces éléments auraient joué en sa faveur pour créer l'association et l'agence de mise en marché, dont il devient le gérant. Il réussit à former une agence de mise en marché en 2003, regroupant 18 boucanières, sept demeurant indépendantes.

L'objet du contrat entre les boucanières vise ainsi la création d'une entreprise de mise en marché. Le contrat initial portait sur le prix de vente des produits et les quotas de production; il prévoyait de fortes amendes en cas de non-respect des conditions de l'entente. Le prix de vente fixé est le même pour tout le monde. Les membres acceptent que tout le poisson soit vendu par l'association, autrement une lourde amende est prévue (150 000 \$). En limitant ainsi la portée du contrat à la vente du produit, les promoteurs du projet voulaient s'assurer l'adhésion du plus grand nombre possible de propriétaires. Ils ont donc écarté initialement une clause sur l'achat du poisson, car plusieurs boucanières entretenaient des rapports privilégiés avec des fournisseurs, qu'ils voulaient naturellement conserver.

Selon un répondant, ce sont les boucanières qui ont défini les règles contractuelles. Le contrat est pris au sérieux, car il prévoit de fortes amendes s'il n'est pas respecté. La vente doit s'opérer par l'entremise de l'association conformément aux stipulations contractuelles. Le respect du contrat apparaît comme une façon de dissiper la méfiance qui existe entre les boucaniers.

La clause portant sur les quotas limitait la production à 50000 boîtes par boucanière ${ }^{17}$. Elle était difficile à accepter pour certains, qui avaient une capacité de production supérieure, mais les membres ont compris que

17. Une boîte équivaut approximativement à huit kilogrammes. 
cet élément de l'entente pouvait être profitable à long terme en obtenant un meilleur prix de vente. C'est d'ailleurs ce qui s'est passé, les prix ayant presque doublé en quelques années (répondant $\mathrm{n}^{\circ} 2$ )

Le gérant promettait que les quotas des boucanières seraient plus élevés la deuxième année. Il demandait que le contrat fût signé pour une durée d'un an avant sa reconduction. Cette stratégie a convaincu plusieurs membres qui voyaient qu'elle ne constituait pas un grand risque, compte tenu de la situation de l'industrie du poisson fumé. Pour certaines boucanières, c'était l'association ou la faillite. D'ailleurs, il semble que celles qui sont demeurées indépendantes réussissaient mieux économiquement :

"On l'essaye pour un an avec une option de deux ans. Pas grand-chose à perdre parce l'industrie était à plat. Ça allait pu pan toute. » (répondant $\left.\mathrm{n}^{\circ} 4\right)$

Au lieu d'être reconduite pour deux ans, l'entente a été renouvelée jusqu'en 2010 et porte désormais autant sur la vente que sur l'achat.

Chaque boucanière doit désormais passer par l'agence de mise en marché pour acheter le poisson, le bois, le sel, bref, les matières nécessaires pour la transformation du poisson. Ce nouveau contrat suppose une modification des perceptions des boucanières. Plutôt que de raisonner simplement en fonction de la qualité de leur produit, ou des liens de confiance qu'elles avaient personnellement développés avec leurs fournisseurs, elles ont peu à peu commencé à raisonner en fonction d'une rationalité collective, voyant que ce n'est plus tant leur réputation personnelle qui comptait, mais la réputation de l'ensemble du groupe. En effet, même si chaque boucanière est identifiée sur les boîtes de produits, c'est d'abord le logo de l'agence de mise en marché qui ressort sur le produit :

"Là ça rentré ceux là qui disiont ben moi, j'ai tout le temps acheté mon poisson à une telle place. Lui a paniqué parce que lui était habitué d'avoir telle qualité, y vendait de tels clients, y savait quosse qui vendait. Asteure, y achète de tout le monde.» (répondant $\left.n^{\circ} 4\right)^{18}$

En outre, la compétition portait aussi sur l'achat des poissons, ce qui faisait monter les prix de la ressource. Or, les boucanières commençaient à constater les avantages qu'il y avait à se regrouper pour lutter contre la concurrence :

«Les plus grosses] gagnont la bataille sur le marché, pis partout sur l'achetage du hareng. Au lieu de se faire éliminer, on va s'mettre ensemble. On va venir plus gros que eux. » (répondant $\mathrm{n}^{\circ} 4$ )

L'association permet notamment de changer le rapport de forces entre les boucanières : «Avant ça, c'tait juste un contre un [...] le plus gros achetait le poisson, pis le petit l'avait pas. Asteure, nous autres, on est là. » (répondant $\left.\mathrm{n}^{\circ} 4\right)$

Les boucanières plus vulnérables voyaient dans l'association un moyen leur permettant de résoudre leurs difficultés financières.

\section{Les conditions de formation de la coopération interentreprises}

\section{Communication et confiance entre les acteurs}

Pour établir un climat de confiance entre les signataires de l'entente, il a fallu instaurer un nouveau mode de communication. Les relations étaient marquées par la méfiance, par une forte compétition, par la peur d'être trompé, par le commérage et par une antipathie entre plusieurs boucanières. Les pratiques de commérage devaient cesser car elles minaient les efforts déployés pour implanter des rapports de réciprocité. Le

18. Nous avons conservé le style oral du parler des Acadiens de cette région généralement qualifiée de chiac. Le "chiac est la variété de français populaire parlée surtout dans le sud-est du NouveauBrunswick, qui se caractérise par l'insertion de mots anglais dans des structures françaises. Il s'agit surtout de mots-noyaux comme so et but, mais aussi d'éléments lexicaux comme watcher" (Boudreau et Gadet, 1998, p. 56). 
gérant incite les membres à régler leurs différends pendant les réunions, plutôt que d'attendre d'être à l'extérieur: "Si vous avez quelque chose à dire, ça se dit en dedans et non pas en dehors. ". Pour $\mathrm{y}$ parvenir, il s'emploie à un travail d'éducation: "Avez-vous pensé ce que je vais penser de vous si vous me parlez en mal de quelqu'un? Je vais me demander : Qu'estce qu'elle dit de moi dans mon dos?» (répondant $\mathrm{n}^{\circ} 1$ ) Cette approche marque un changement par rapport aux relations qui prévalaient auparavant. «[...] le gros problème à Cap-Pelé, c'était d'la jalouserie. » (répondant $\mathrm{n}^{\circ} 7$ ). Ainsi, « la plupart du monde s'parlait, mais [...] pas de confiance en toute un l'autre. » (répondant $\mathrm{n}^{\circ} 7$ )

Le fait de participer régulièrement à des réunions incite les membres du groupe à communiquer entre eux : «Les premières réunions de l'association, ça se parlait pas trop. Pis là, ça v'nu à mesure que, c'est ça, beaucoup de réunions, t'es beaucoup ensemble, tu fais une p'tite joke, pis là, ça commence à venir mieux. Même que ça v'nu que les gars ont confiance l'un à l'autre. » (répondant $\mathrm{n}^{\circ} 10$ )

Peu à peu, des liens de confiance se créent, contribuant à former un capital social pouvant s'avérer souvent utile. Ce qui contribue à développer des relations de confiance n'est pas seulement une question d'éducation et de bonne volonté. Le fait que chaque boucanière sait à l'avance quelle quantité elle produira, quelle quantité l'autre boucanière produira et que les propriétaires vendront leur produit au même prix constitue un facteur objectif qui contribue à mettre fin à la compétition : "On a vu qu'on a [...] pu mettre des quotas, pis là, après ça, arrêter la jalouserie entre les boucaneux. » (répondant $\mathrm{n}^{\circ} 7$ )

\section{La coopération et la réduction de l'incertitude}

La passation d'un contrat associant les boucanières à une entreprise de mise en marché a permis de revoir l'organisation de la production. Chaque boucanière sait à l'avance quelle sera sa production annuelle du fait de l'existence de quotas. Par ailleurs, elle est assurée d'écouler sa production à un prix qui sera le même que celui de ses anciens concurrents. Pour les boucanières, il n'est plus nécessaire de produire rapidement pour obtenir les meilleurs prix ou pour s'assurer de vendre ses produits. Le promoteur du projet a mis en place un système de planification selon lequel, peu importe le moment où la boucanière est prête à écouler sa production, elle est assurée de pouvoir le faire. "Aujourd'hui, y aviont plus ce tracaslà [...] y pouvont faire un budget asteure [...] avant ça, le monde vivait de jour en jour, pis y savaient jamais. » (répondant $\mathrm{n}^{\circ} 1$ )

Ce système de planification réduit grandement l'incertitude pour les boucanières, au sujet notamment du prix de vente et de la possibilité de vendre. "Quand même que les prix changeraient, à la fin de l'année, tout le monde a tout le même [prix]. » (répondant $\mathrm{n}^{\circ} 1$ ) En outre, il permet de produire un poisson de meilleure qualité : "La qualité du poisson à cause de l'horloge, y faisont le meilleur. » (répondant $\mathrm{n}^{\circ} 1$ ) L'horloge est le nom donné au système de gestion de la production qui assure à chacun d'écouler sa production quand il sera prêt. Voici, par contraste, un extrait qui illustre la situation antérieure : «Si tu mets un groupe, pis qu'y met un prix, on va dire 10 piastres la boîte [...] ben l'autre, lui, peut venir, pis mettre 9 1/2 lui, pis lui va vendre plus vite. C'tait tout le temps d'même que ça allait. » (répondant $\mathrm{n}^{\circ}$ 5) $\mathrm{La}$ compétition défavorisait les boucanières tant à la vente des produits marins qu'à l'achat des produits nécessaires à la transformation. Elle s'exerçait donc contre l'industrie en amont et en aval, au point de menacer l'existence de plusieurs entreprises.

Avec l'agence de mise en marché, les boucanières contrôlent plus l'achat et la vente de produits marins. Plus d'une vingtaine de comités auxquels siègent plusieurs membres permettent de gérer un ensemble d'activités au sein de la nouvelle entreprise. 
Ces comités reposent sur l'engagement des membres de l'association, qui prennent ainsi en charge les tâches financières, le transport, la gestion de la production (quotas), l'achat de sel et de bois, les relations avec les membres, les licences, les questions juridiques et la qualité du poisson. La présence aux comités de membres provenant de plusieurs boucanières contribue à accroître leur confiance entre eux. Le gérant est informé des activités de chacun des comités, sur lequel il a droit de regard. Ces comités ne prennent pas de décision. Ils exécutent un travail en fonction des décisions qui ont été prises ou ils préparent des questions en vue de les présenter aux membres décideurs. «Aucune décision sort du comité [exécutif] sans que ça été passé aux membres." (répondant $\mathrm{n}^{\circ} 1$ )

\section{La formation d'un groupe social}

Pour parvenir à former une association de boucanières qui se concrétise dans un contrat et dans la création d'une agence de mise en marché, il a fallu passer d'une situation où chaque boucanière travaillait pour ellemême à une situation où toutes travaillent de concert pour une entreprise de mise en marché qui les sert en retour. Ce passage est facilité par la mise en place de l'agence, qui s'accompagne d'une série de règles dont le respect par les boucanières leur permet de poursuivre leur intérêt tout en le réconciliant avec celui du collectif qu'il forme désormais. Autrement dit, les conditions qui accompagnent la création de l'agence de mise en marché et auxquelles doivent se soumettre les boucanières contribuent à éliminer la compétition qu'elles se livraient. Par la mise en place de certaines conditions qui règlent les échanges entre les boucanières, en leur garantissant notamment la vente de leur produit aux mêmes prix, les signataires de l'entente substituent aux relations compétitives des relations de coopération. L'agence constitue ainsi un espace social de coopération au lieu de l'espace marchand antérieur et assure la transition vers une CIE.
Nous avons vu le rôle qu'exerce le contrat dans cette transition. Il joue un rôle incitatif central dans l'orientation des comportements des boucanières. Cependant, la sanction tout comme l'intérêt ne suffisent pas à expliquer le fonctionnement de cette CIE. Des facteurs symboliques doivent favoriser le développement d'un sentiment d'appartenance. Le groupe de boucanières se forme et existe en fonction d'éléments symboliques qui l'expriment et l'identifient et qui donnent corps au projet. L'existence du groupe s'exprime notamment dans l'emploi de symboles d'identification, qui constitue un facteur déterminant dans sa formation. L'adoption d'un logo unique pour représenter le groupe est une action qui exprime la reconnaissance des membres de l'association et leur engagement à lui donner une existence concrète. Pour certains, il n'a pas été facile d'adopter un logo unique, car cela signifiait que la réputation qu'ils avaient construite et l'image avec laquelle ils s'étaient fait connaître depuis des années se trouvaient diluées dans la nouvelle entité. "Nous autres, ça fait 50 ans qu'on vend sous [nom de l'entreprise], pis les gens làbas savent que [...] c'est une belle qualité. » (répondant $\mathrm{n}^{\circ}$ 4) Cependant, ils acceptent d'adopter le logo, car ils croient au projet d'association.

Par ailleurs, le gérant décide de créer des pièces de vêtement (casquette, manteau ou T-shirt) aux couleurs de l'association et encourage les membres à les porter lors des rencontres et des activités de représentation. "À deux heures, je veux tous vous voir icitte. Mettez vos swetshirt - ils ont toutes leur swetshirt- toutes leurs casques avec le logo. » (répondant $\mathrm{n}^{\circ} 1$ )

Pour donner naissance socialement au groupe, un échange symbolique se produit entre le promoteur et les membres du groupe. D'une part, les propriétaires de boucanières tendent à faire reposer l'existence de l'association sur l'action du gérant, qui, en retour, fait reposer l'existence et le fonctionnement du groupe sur ses membres. 
L'extrait qui suit illustre la perspective du gérant : "Aujourd'hui personne runne [dirige]. Moi je ne runne pas, c'est toute la gang qui runne. » (répondant $\mathrm{n}^{\circ} 1$ ) Lorsque l'entreprise de mise en marché reçoit un représentant du gouvernement provincial, le gérant veut lui présenter "la personne la plus importante du groupe » et présente chacun des propriétaires des boucanières, qui sont autour de la table, signifiant ainsi que c'est le groupe qui est au centre de l'entreprise.

Cependant, pour les signataires de l'entente, c'est grâce aux qualités personnelles du gérant que le groupe existe et fonctionne. Plusieurs commentaires soulignent ses qualités : "Il a un don, j'peux pas dire. Il écoute beaucoup. Il lit le monde. » (répondants $n^{\text {os }} 2$ et 3 ) On souligne ainsi sa capacité à comprendre les personnes en les écoutant. Son sens de l'écoute lui a servi à saisir les enjeux entre les boucanières. On souligne sa connaissance de la nature humaine (répondant $\mathrm{n}^{\circ} 7$ ), de même que sa capacité d'analyse afin de comprendre la source des conflits et de bien connaître les propriétaires des boucanières (répondants $n^{\text {os }} 1$ et 4 ) pour proposer des solutions (répondants $\mathrm{n}^{\text {os }} 4,5$ et 7). Certains expliquent la création de l'association par l'action du gérant (répondant $\mathrm{n}^{\circ} 8$ ) : "J'parle beaucoup $d u$ gérant parce que c'est toute à cause de lui [...] y aviont confiance en lui. » (répondant $\mathrm{n}^{\circ} 7$ ) Le fait que le gérant provient d'un milieu extérieur aux boucanières les a aidées à lui faire confiance. (répondant $\mathrm{n}^{\circ} 4$ et $\mathrm{n}^{\circ} 6$ )

Le gérant insiste pour que les signataires de l'entente «tournent la page du passé » (répondant $\mathrm{n}^{\circ} 2$ ), oublient querelles et conflits. Il propose une nouvelle façon de communiquer, en encourageant la franchise et en invitant les membres à exprimer ouvertement dans les assemblées ce qui fait l'objet d'un conflit ou d'un désaccord.

Une autre qualité soulignée chez lui est qu'il sait mener son action progressivement en dépassant la méfiance naturelle et les antipathies. "Si on l'avait passé [le règle- ment sur les quotas] quand tous les membres sont ici, ça aurait pas marché. » (répondant $n^{\circ}$ 1) À cette fin, il consulte d'abord les membres un à un, au lieu de les mettre tous dans la même pièce au début des discussions. (répondant $n^{\circ} 4$ ) De cette façon, il reste au centre des relations entre les membres et a accès à toute l'information nécessaire pour gérer les différends et favoriser des actions compatibles avec les solutions pressenties. S'il y a des membres qui résistent à un projet, alors on tente de le convaincre : "Si quelqu'un était pas d'accord, là on travaillait sur lui. » (répondant $\mathrm{n}^{\circ} 2$ ).

Le gérant tient à ce que la parole donnée et les ententes conclues soient respectées. Par exemple, les boucanières devaient respecter un délai pour signer leur contrat. Un propriétaire a voulu signer l'entente après le délai fixé et il a été refusé. Cette façon de faire envoyait aux autres le message que ce qui est convenu doit être respecté, mettant fin ainsi à certaines pratiques informelles d'arrangements.

La constitution du groupe se fonde sur un don et un contre-don symbolique entre les propriétaires de boucanières et le gérant de l'agence de mise en marché. Alors que le groupe projette ses capacités organisationnelles et son savoir-faire sur le gérant du groupe, celui-ci leur retourne symboliquement cette capacité. Cet échange symbolique sous-tend un rapport de pouvoir entre le gérant et les propriétaires de boucanières, à l'avantage du gérant qui a ainsi les coudées franches pour diriger cette association. Par ailleurs, la formation du groupe exige l'acquisition de nouvelles compétences et d'un nouveau savoir-faire d'ordre organisationnel. Le gérant veut que le groupe «tourne la page » et « allume », c'est-à-dire que ses membres comprennent l'importance de changer leurs relations pour que l'association fonctionne. Lorsqu' on demande à des répondants ce qui arriverait si le gérant démissionnait, on nous répond : "Il a promis que quand il va partir, qu'on serait prêt à prendre 
la relève. » (répondant $\mathrm{n}^{\circ} 2$ ) Progressivement, les propriétaires développent leurs capacités organisationnelles et, selon le gérant, pourront prendre en charge leur association. Pour se former, cette dernière a besoin d'une personne extérieure, aux qualités charismatiques, conférées par les propriétaires de boucanières, qui projettent sur elle leur capacité de se prendre en charge.

\section{Le passage d'une rationalité individualiste à une rationalité collective}

L'existence du groupe repose sur le passage d'une logique individualiste à une logique collective de l'intérêt. Dans le cadre de l'association, chaque membre continue de poursuivre son intérêt personnel, mais il le fait en considérant désormais l'intérêt du groupe, sa réussite personnelle étant subordonnée à celle du groupe. Par ailleurs, l'avantage de former un groupe face aux boucanières indépendantes motive les boucanières à maintenir leur effort associatif : «À cause d'la compétition qu'on a encore, parce qu'elle est plus féroce, hein? Avant ça, c'tait juste un contre un, le plus gros achetait le poisson, pis le petit l'avait pas. Asteure, nous autres, on est là. » (répondant $\mathrm{n}^{\circ} 4$ )

Le gérant fait néanmoins un travail de sensibilisation pour encourager la mise en place d'une logique d'action collective et solidaire parmi les membres du groupe : «Pis moi, faut que j’te protège [montre quelqu'un] puis faut que j'te protège [montre quelqu' un d'autre] [...] sans ça, moi j'peux pas rien faire. J'ai besoin de toi. » (répondant $\mathrm{n}^{\circ}$ 1) Cet extrait montre que le passage d'une rationalité individualiste à une rationalité collective passe par un apprentissage grâce auquel chacun comprend que son sort est lié à celui de son partenaire. Il semble que le travail de sensibilisation a porté fruit : «Telle personne qu'a besoin de l'aide, on va l'aider. Ça fait pas de différence qui c'est, quelqu'un a besoin de l'aide, on va l'aider. Avant ça, tu voyais pas ça. » (répondant $n^{\circ} 7$ ) L'exemple d'un comportement tourné vers le succès du groupe viendrait d'ailleurs du gérant : «Puis lui, il a fait des gros sacrifices pour nous autres. On va y rendre ça. » (répondant $\mathrm{n}^{\circ} 4$ ) Un sentiment de dette s'exprime ainsi envers le gérant.

Pour amener les membres à adopter une approche collective, le gérant encourage la formation d'un sentiment d'égalité entre eux en leur faisant abandonner l'esprit de compétition. Il affirme : «C'est pas la grandeur de la boucanière qui compte [...] en dedans, c'est pareil, c'est noir. » (répondant $\mathrm{n}^{\circ} 1$ ), insistant ainsi sur ce que les boucanières ont en commun.

\section{L'intérêt pour une association}

Le groupe est en meilleure position pour négocier l'achat des ressources requises pour la production et la vente des produits marins. Il peut acheter en plus grande quantité à des prix avantageux. Son pouvoir de négociation s'est accru grandement grâce à l'association des 18 boucanières. Les avantages de la solidarité que permet le groupe ont pu être démontrés dans d'autres situations où, auparavant, certains acteurs extérieurs pouvaient profiter des dissensions et de la compétition entre les boucanières, ce qui constituait des facteurs d'affaiblissement des activités.

Dans cet exemple, nous voyons le groupe intervenir à un palier politique pour bloquer la délivrance d'un nouveau permis. Une boucanière indépendante a voulu acheter la licence d'une boucanière qui avait cessé ses activités afin d'augmenter sa production. Elle a présenté une requête à un organisme gouvernemental, mais le groupe s'est rendu à Fredericton pour s'y opposer (répondant $\mathrm{n}^{\circ} 6$ ) Le gérant organise donc une rencontre avec un représentant du gouvernement pour contrer cette décision :

Avant, j'étais seule. J'aurais rien pu faire [pour empêcher la délivrance d'un nouveau permis] [...] Si moi j'fais une décision, mais que j'suis toute seule, j'suis toute seule, mais si on est 18... Là, ils voient [le gouvernement] qu'on ne peut plus s'amuser avec Cap-Pelé Herring. (répondant $\mathrm{n}^{\circ} 2$ ) 
Plusieurs observateurs de l'industrie considèrent qu'une des causes de ses difficultés est la surproduction, ce qui a pour effet de faire diminuer les prix. Or, certains répondants affirment que le jeu politique peut influencer des décisions favorables à certaines boucanières. Les décisions politiques semblent moins reposer sur une analyse objective de l'industrie que sur des sympathies politiques. "Lui, y avont acheté [la boucanière] en cachette en travers de politique. La politique là, j'sais comment ça travaille, moi, pas mal. » (répondant $\mathrm{n}^{\circ}$ 6) Le rapport réalisé par la société conseil Co-fish Consultants rappelait déjà en 1985 la responsabilité du gouvernement dans la structuration et le développement de l'industrie.

Une autre situation a permis de démontrer la force de la solidarité pour le groupe. Au début de la formation de l'association, un acheteur porte plainte au sujet de la qualité du produit. Auparavant, il était difficile d'argumenter. Cette façon de faire constituait une stratégie habituelle des acheteurs pour faire baisser les prix. Cette stratégie fonctionnait alors que chaque boucanière était isolée. Cette fois cependant, plusieurs boucanières décident d'aller rencontrer les acheteurs sur place pour discuter de la qualité du produit. «Un claim [réclamation] ? sept-huit de s'eux ont monté [en République dominicaine], tout a été fini. On est $18 \ldots »$ (répondant $\mathrm{n}^{\circ}$ 2) Cette stratégie des acheteurs s'est avérée infructueuse et n'a plus été employée par la suite.

Le fait que les boucanières se parlent et se concertent davantage a permis également d'éliminer une autre pratique courante. Les boucanières connaissaient un roulement de personnel élevé. Plusieurs employés profitaient également de la situation qui existait entre elles. Étant donné que peu de boucanières entretenaient des liens de communication entre elles, les employés allaient au plus offrant. Cette pratique n'est plus possible grâce à la concertation entre les boucanières membres de l'association. Les membres de l'association constatent donc les avantages qu'offre le groupe : La plupart du monde s'parlait, mais [...] pas de confiance en toute, un l'autre. Asteure, y a une grande confiance entre les boucaneurs. À cause du groupe tout ensemble, y a un contrôle. (répondant $\mathrm{n}^{\circ} 7$ )

Ils sont conscients du fait que les acteurs extérieurs ne peuvent plus profiter des dissensions et de la compétition qui prévalaient.

\section{Conclusion}

Aux yeux de ses membres, la création d'une agence de mise en marché constitue une réussite puisque, pour la première fois, on parvient à créer une association entre les entreprises qui, en outre, donne des résultats positifs. La constitution de l'association a permis à certains propriétaires de surmonter leurs difficultés économiques et à leurs boucanières de prendre de la valeur : Ça $a$ monté la valeur [...] ça icitte [désigne la boucanière] [...] Juste à cause du Logo, juste à cause de la compagnie. Tu pouvais pas la donner. Y pouviont pas la laisser à leur garçon [...] Ça a plus avancé dans les derniers trois ans que dans les derniers 50. Mon père a boucané toute sa vie, pis y a pas d'argent (répondant $\mathrm{n}^{\circ} 4$ )

Cependant, d'aucuns pourront penser que la création de l'association n'a fait que déplacer un vieux problème. La compétition et les relations conflictuelles se vivent maintenant entre les boucanières indépendantes et celles qui sont membres de l'association. D'ailleurs, un répondant travaillant dans une boucanière indépendante mentionne que, depuis la création de l'association, les indépendants se concertent davantage. $\mathrm{La}$ compétition en matière d'achat et de vente est âpre entre les indépendants et l'association, cette dernière profitant de son poids pour obtenir des arrangements qui lui sont favorables, voire exclusifs. Si au début les indépendants ont profité de la hausse et de la stabilité des prix engendrés par l'existence de quotas, la compétition a repris de plus belle, mais, cette fois, entre les deux clans de boucanières. Les pratiques de commérage visant à porter atteinte à la réputation 
de l'un ou de l'autre clan se sont également déplacées et se concentrent maintenant sur le clan adverse. Cette relation entre l'association des boucanières et les entreprises qui sont demeurées indépendantes pourrait faire l'objet d'une autre étude.

Notre étude s'est proposée au premier chef de mettre en évidence les éléments constitutifs d'un mode de coopération entre des entreprises auparavant en forte concurrence. Plusieurs facteurs nous permettent de comprendre le passage opéré entre des rapports de méfiance et des rapports de confiance réciproques, contribuant à la formation d'un nouveau capital social : - l'intérêt des boucanières en difficultés à trouver une solution pour sortir de l'impasse dans laquelle les confinait la situation du marché ;

- l'intervention d'une personne étrangère à l'industrie et au milieu ;

- l'apprentissage d'un mode de communication fondé sur la transparence, le faceà-face et la franchise ;

- l'accès à l'information et le contrôle de la diffusion de l'information par le responsable en ce qui concerne la mise en œuvre de nouveaux projets ;

- l'utilisation de symboles désignant le groupe formé par la CIE et cristallisant un nouveau sentiment d'appartenance et - la passation d'un contrat officialisant les conditions de l'entente.

L'historique des acteurs est souvent garant d'un capital de confiance ou non. "Past experience, future expectations and networks embeddedness create initial trust and allow for implicit threats ». (Blumberg, $2001)^{19}$. Dans notre cas, l'histoire des boucanières les a conduits tout droit à une situation de méfiance mutuelle plutôt que de confiance. Nous avons vu l'insistance avec laquelle le gérant demande aux membres de rompre avec le passé pour mettre en place un esprit de coopération.

19. In Blumberg, page 830.
La constatation des avantages qu'offre la coopération motive les propriétaires à demeurer dans l'association. Parmi ces avantages, mentionnons :

- les avantages économiques de la vente et de l'achat en groupe ;

- le rapport de force que permet d'instaurer la solidarité entre les boucanières auprès des autres boucanières indépendantes, des employés, des acheteurs et auprès du gouvernement ainsi que

- la stabilité de la planification et la réduction de l'incertitude que permet la réduction des rapports de compétition entre les boucanières.

Comme le mentionne Loup (2003), la réduction de l'incertitude représente un élément central de la CIE : «Cette situation de groupe permet de mieux maîtriser l'environnement et en particulier les marchés de sorte que la vulnérabilité et l'incertitude soient réduites. $»^{20}$

Plusieurs facteurs ont donc concouru à former un mode de CIE, mais ce qui demeure au fondement de cette forme de coopération est l'échange symbolique entre le responsable de l'association d'entreprises et les membres de celle-ci. En effet, le groupe fait reposer son pouvoir constitutif sur une personne qui apparaît comme le socle sur lequel il s'érige, alors que cette personne retourne à ses membres ce pouvoir constitutif du groupe. La nature des rapports sociaux qui sont à la base de la CIE se dissimule ainsi à la conscience des membres du groupe, mais c'est ce qui confère une certaine légitimité au pouvoir exercé par le gérant et une capacité d'assurer la cohésion du groupe. Comme le dit Marx (1982) : « [...] les hommes et leurs rapports nous apparaissent placés la tête en bas comme dans une "camera obscura" ${ }^{21}$. Autrement dit, c'est par ce processus que des propriétaires de boucanières parviennent à former une association.

20. In Loup, page 197.

21. In Marx, page 78. 
La mesure où la capacité de former un groupe est retournée à ses membres que ceuxci pourront éventuellement acquérir les capacités nécessaires pour se prendre en charge.

Il s'agit, bien entendu, d'une modalité particulière, contingente, entourant la formation d'une CIE. D'autres modalités sont possibles, mais ce que montre notre analyse est la nécessité de recourir à des facteurs sociaux (les normes, les symboles, la confiance, le contrat, sans oublier l'intérêt) pour comprendre la CIE, dont le succès repose sur la construction d'un groupe social proprement dit. En d'autres termes, l'organisation doit produire du social, voire du capital social, et, à cette fin, plusieurs modifications sont exigées pour faire passer les membres d'une forme de régulation marchande à une forme de régulation associative reposant sur un contrat, la confiance et un accord négocié entre des acteurs $(\text { Devel })^{22}$.

Pour le dire dans les termes de Granovetter (1994), nous dépassons ainsi la forme sous-socialisée de l'individu poursuivant son intérêt personnel pour parvenir à une situation socialisée dans laquelle l'action individuelle est désormais intégrée dans le collectif que forment désormais les boucanières. C'est en ce sens que les boucanières parviennent à produire du social et que nous pouvons parler d'encastrement social des pratiques économiques. Cependant, avant de souhaiter l'intégration sociale des activités économiques, il est opportun de se demander s'il existe bien une communauté dans laquelle peut s'insérer l'action individuelle. Comme le montre notre cas, la communauté est parfois d'abord à construire. La confiance qui en découle s'appuie sur les règles de réciprocité qui sont contenues dans le contrat au fondement du collectif. Avant de constituer un facteur déterminant

22. Devel T. «Peut-on gouverner l'Internet? Une perspective démocratique », [en ligne] http://c2so.ens-lsh.fr/IMG/pdf/23-COMMINT__Thiery_Vedel.pdf (consulté le 17 juin 2008). des activités économiques, la confiance doit se construire.

Mentionnons, pour finir, que les gestionnaires et les intervenants qui souhaitent tabler sur le capital social, voire le développer pour créer des associations ou des alliances entre des entreprises doivent réfléchir à la composante émergente et à la complexité du social qui échappent à toute approche instrumentale. Comme nous le rappelle Godbout (2000) «le lien social n'existe vraiment que lorsqu'il n'est pas un moyen, lorsqu'il est voulu pour lui-même $»^{23}$. Un tel projet de CIE a plus de chance de réussir s'il donne lieu à un nouvel espace social où se forment des liens sociaux véritables entre les membres de l'association.

Le questionnement sur l'intégration sociale des activités économiques connaît un regain d'intérêt grâce à la nouvelle sociologie économique, comme en témoignent les nombreux écrits sur le sujet (voir Lévesque et al., op. cit.). Contrairement à la conception économiste, cette littérature tend à montrer l'intégration de l'économie dans la société comme un fait social (Orléans, 2004), tout en faisant généralement l'économie d'une réflexion sur l'existence et la forme de la société dans laquelle l'économie s'intègre. Dans le contexte de la mondialisation, du déclin de la communauté (Ferry, 2001), de l'atomisation sociale et de l'individualisme exacerbé, demandons-nous, d'abord, si communauté il y a, et ensuite, quelle est sa forme, puisque l'intégration de l'économie dans une société atomisée par la structuration marchande de la culture ne rejoint guère le projet d'une économie sociale préconisée par plusieurs observateurs et acteurs. D'autant plus que les valeurs de la compétition, de la concurrence et de l'intérêt personnel sont devenues, selon plusieurs témoins de la culture occidentale contemporaine, prédominantes (Maréchal, 2000).

23. In Godbout, page 299. 


\section{RÉFÉRENCES BIBLIOGRAPHIQUES}

Avadikyan A., Llerena P., Matt M., Rozan A., Wolff S. (2001). Organisational rules, codification and knowledge creation in inter-organisation cooperative agreements. Research policy, $\mathrm{n}^{\circ} 30$, p. 1443-1458.

Bellon B., Niosi J. (2001). Institutionalist Theories and Inter-Firm Alliances: The Transaction Cost, Competence and Evolutionary Debate Revisited. Current sociology, vol. $49, \mathrm{n}^{\circ} 4$, p. 67-84.

Blumberg B.-F. (2001). Cooperation Contracts Between Embeded. Organization studies, vol. 22, n ${ }^{\circ} 5$, p. 825-852.

Boudreau A., Gadet F. (1998). Attitudes en situation minoritaire. L'exemple de 1'Acadie. Le français en Afrique, Revue du Réseau des Observatoires du Français Contemporain en Afrique Noire, $\mathrm{n}^{\circ} 12$, p. 55-61.

Brun R., LeBlanc R.-G. (2005). Histoire de Cap-Pelé, 1804-2004: le passé d'une communauté dynamique en Acadie. CapPelé (Nouveau-Brunswick.).

Co-fish Consultants Ltd. (1985). The Smoked Herring Industry, a detailed study for Cap-Pelé Bloater Smokers' Association. Moncton (Nouveau-Brunswick).

Farrell H. (2005). Trust and political economy. Comparative Political Studies, vol. $38, n^{\circ} 5$, p. 459-483.

Ferry J.-M. (2001). De la Civilisation. Civilité, Légalité, Publicité. Paris, Éditions du Cerf.

Forgues É. (2007). Du conflit au compromis linguistique, L'État et le développement des communautés francophones en situation minoritaire. Rapport de l'ICRML, Moncton [en ligne] www.icrml.ca.

Forgues É. (2004). Capital social, gouvernance et rationalisation des pratiques communautaires, Outils théoriques et méthodologiques. Rapport de l'ICRML,
Moncton [en ligne] www.icrml.ca.

Godbout J.-T. (2000). L'engagement : une fidélité. Sociologie du travail, $\mathrm{n}^{\circ} 42$, p. 289- 300.

Granovetter M. (1994). Les Institutions Économiques comme Constructions Sociales : un Cadre d'Analyse. In André Orléans (Dir.), «Analyse Économique des Conventions », PUF, p. 79-94.

Granovetter M. (1985). Economic action and social structure: the problem of embeddedness. American Journal of Sociology, vol. 91, n 3, p. 481-510.

Granovetter M. (1973). The strenght of weak ties. American Journal of Sociology, vol. 78, $\mathrm{n}^{\circ}$ 6, p. 1360-1380.

Grewe N.-R. (2003). Social capital and local development: an exploration of three forms of community-based social capital. Iowa State University, Faculty of philosophy, Doctoral Dissertation.

Guillouzo R., Thépaut Y. (2004). Une interprétation de la coopération interentreprises en termes de pouvoir informationnel. La revue des Sciences de Gestion, vol. 39, n 206, p. 41-60.

Judge R. (2003). Le capital social, établir les fondements de la recherche et de l'élaboration de politiques. Horizons 6(3), p. 7-12.

Laville J.-L., Sainsaulieu R. (1997). Sociologie de l'association; des organisations à l'épreuve du changement social. Paris, Desclée De Brouwer.

LeBlanc B. (2000). New Technology in the Smoked Herring Industry, South-East New Brunswick. Dieppe (NouveauBrunswick), avril.

Lévesque B., Bourque G.-L., Forgues É. (2001). La nouvelle sociologie économique. Paris, Desclée de Brouwer.

Loup S. (2003). Les petites entreprises des métiers d'art. Revue française de gestion, vol. 29, n 144, p. 195-209. 
Maréchal J.-P. (2000). Humaniser l'économie. Paris, Desclée de Brouwer.

Marx K.(1982). L'idéologie allemande. Paris, Éd. sociales.

Mubangizi B.-C. (2003). Drawing on social capital for community economic development: Insights from a South African rural community. Community Development Journal 38(2), p. 140-150.

Orléans A. (2004). Réflexion sur les fondements institutionnels de l'objectivité marchande. Cahiers d'économie politique, tome 44, p. 181-196.

Pêches et Océans Canada (1987). L'impact économique de l'industrie du hareng fumé du sud-est du Nouveau-Brunswick sur la région étudiée. Division de l'analyse économique, Région du Golfe, Moncton (Nouveau-Brunswick).

Ponthieux S. (2003). Que faire du « social capital »? Département des prix à la consommation, des ressources et des conditions de vie des ménages. Direction des statistiques démographiques et sociales, Institut national de la statistique et des études économiques.

Sainsaulieu R. (1992). L'entreprise, une affaire de société. Paris, Presses de Sciences Po.

Yami S. (2003). Petite entreprise et stratégie collective de filières. Revue française de gestion, vol. 29, n 144, p. 165-179. 